\section{Vorhersagewert, positiver}

R.-D. Hilgers ${ }^{1}$, N. Heussen ${ }^{1}$ und S. Stanzel ${ }^{2}$

${ }^{1}$ Institut für Medizinische Statistik, Universitätsklinikum der RWTH Aachen, Aachen, Deutschland

${ }^{2}$ DKFZ Heidelberg, Heidelberg, Deutschland

Synonym(e) A-posteriori-Wahrscheinlichkeit; Prädiktiver Wert des positiven Testresultats;

Englischer Begriff positive predictive value

Definition Der positive Vorhersagewert gibt die (bedingte) Wahrscheinlichkeit an, krank zu sein, falls ein positives Testergebnis vorliegt.

Beschreibung Der positive Vorhersagewert wird geschätzt durch den Quotienten aus der Zahl der Erkrankten mit positivem Test und der Gesamtzahl der testpositiven Fälle (d. h. durch den Quotienten $\mathrm{a} /(\mathrm{a}+\mathrm{b})$; Bezeichnungen s. Tabelle im Eintrag $>$ Vierfeldertafel).

Da der positive Vorhersagewert die diagnostische Fähigkeit eines positiven Testergebnisses widerspiegelt, wird er zuweilen auch als A-posteriori-Wahrscheinlichkeit für das Auftreten einer Erkrankung bezeichnet. Sind > Prävalenz (prev), Sensitivität (Se; > Sensitivität, diagnostische) und Spezifität (Sp; > Spezifität, diagnostische) eines Testverfahrens bekannt, lässt sich der positive Vorhersagewert mittels des Satzes von Bayes ( $\vee$ Bayes, Satz von) berechnen:

$$
\mathrm{PV}^{+}=\frac{\mathrm{Se} \times \text { prev }}{\mathrm{Se} \times \text { prev }+(1-\mathrm{Sp}) \times(1-\text { prev })}
$$

Anhand dieses Zusammenhangs lässt sich erkennen, dass der positive Vorhersagewert bei zunehmender Prävalenz steigt bzw. dass der positive Vorhersagewert bei hoher Spezifität ebenfalls hoch ist. Dieser Zusammenhang bedingt, dass bei der Anwendung eines diagnostischen Tests $(\triangleright$ Test, diagnostischer) in einem Risikokollektiv höhere positive Vorhersagewerte zu erwarten sind.

\section{Literatur}

Hilgers R-D, Bauer P, Scheiber V (2002) Einführung in die Medizinische Statistik. Springer, Berlin/Heidelberg/New York 